\title{
Glucose-responsive polymer brushes for microcantilever sensing $\dagger$ :
}

\author{
Tao Chen, ${ }^{a}$ Debby P. Chang, ${ }^{a}$ Ting Liu, $^{b}$ Ramya Desikan, ${ }^{c}$ Ram Datar, ${ }^{d}$ Thomas Thundat, $^{c}$ Rüdiger Berger ${ }^{b}$ \\ and Stefan Zauscher $* a$
}

\author{
Received 4th December 2009, Accepted 8th February 2010 \\ First published as an Advance Article on the web 23rd February 2010 \\ DOI: $10.1039 / b 925583 d$
}

\begin{abstract}
Glucose responsive polymer brushes were synthesized on gold substrates and microcantilever arrays. The response properties of these brushes were evaluated by exposing them to different glucose concentrations for a range of $\mathrm{pH}$ values. This work demonstrates the potential for polymer brush-functionalized micromechanical cantilevers as glucose detectors. Furthermore, the work demonstrates that stimulus-responsive polymer brushes on micromechanical cantilevers have a significantly larger bending response due to glucose binding compared with self-assembled monolayers.
\end{abstract}

There is considerable interest in micromechanical cantilevers grafted with stimulus-responsive polymer brushes for sensing applications in aqueous environments. ${ }^{1-7}$ These micromechancial cantilever sensors provide, due to conformational changes in the polymer brush, a much larger bending deflection in response to changes in temperature, light, chemical environment, and $\mathrm{pH}$, compared to cantilevers decorated with self-assembled monolayers (SAMs), where conformational changes are limited. Furthermore, polymer brushes can be synthesized to carry several functional groups and allow thus tuning of the bending response.

Poly $(N$-isopropylacrylamide) (PNIPAAM) has a lower critical solution temperature (LCST) of about $32{ }^{\circ} \mathrm{C}$ in pure water, and can be triggered by temperature and co-solvents to respond with a reversible de-swelling phase transition when grafted to surfaces. ${ }^{8-11}$ To engineer the sensitivity to specific stimuli, functional monomers can be incorporated into polymer brushes via copolymerization, and functional moieties can be introduced through subsequent chemical modification. ${ }^{12}$ Furthermore, our recent work has shown that micromechancial cantilevers, decorated on one side with a PNIPAAM brush or a poly( $N$-isopropylacrylamide- $c o-N$-vinylimidazole) (PNIPAAM-PVI) brush, offered a simple way to detect changes in solvent type, temperature, and $\mathrm{pH}$, promising great potential for sensing applications in micro-fluidic devices. ${ }^{7}$

Of particular interest for the biomedical field is the transduction of a biochemical stimulus. ${ }^{6,13}$ An important target molecule is glucose because it is a source of energy and a metabolic intermediate for living

${ }^{a}$ Center for Biologically Inspired Materials and Materials Systems, and Department of Mechanical Engineering and Materials Science, Duke University,Durham, NC,27708, USA. E-mail: zauscher@duke.edu

${ }^{b}$ Max Planck Institute for Polymer Research, Mainz, 55128, Germany

'Oak Ridge National Laboratory, Oak Ridge, TN, 37831, USA

${ }^{d}$ Biomedical Nanoscience Institute, University of Miami, Miami, FL, 33136, USA

$\dagger$ This paper is part of a Journal of Materials Chemistry themed issue on Actively Moving Polymers. Guest editor: Andreas Lendlein.

* Electronic supplementary information (ESI) available: Details of materials, polymerization, fabrication and characterization. See DOI: $10.1039 / \mathrm{b} 925583 \mathrm{~d}$ cells, and because of the widespread disease of diabetes that requires monitoring of blood sugar levels. ${ }^{14} \mathrm{~A}$ micromechancial cantilever, functionalized with a glucose-responsive polymer brush may provide a means to quantify glucose concentrations even in complex physiological mixtures. As a first step to produce such a system, we developed a copolymer brush complex with the ability for biomolecular recognition and whose response magnitude can be tuned, to detect and transduce the concentration of glucose (or other diols) in the brush environment. It is well known that phenylboronic acid (PBA) can bind diols through reversible boronate ester formation, ${ }^{15}$ and incorporation of boronic acid into linear copolymers, ${ }^{16-18}$ latex ${ }^{19}$ and polymer gels ${ }^{20-22}$ has been demonstrated. Here we report the synthesis of glucose-responsive poly( $N$-isopropylacrylamide)-copoly(acrylic acid)-(3-aminophenyl-boronic acid) (PNIPAAM-coPAA-PBA) brushes, and explore their potential for microcantilever based detection of glucose at physiologically relevant concentrations.

Fig. 1 schematically shows our synthesis approach and subsequent glucose binding. In the first step, we prepared micropatterned

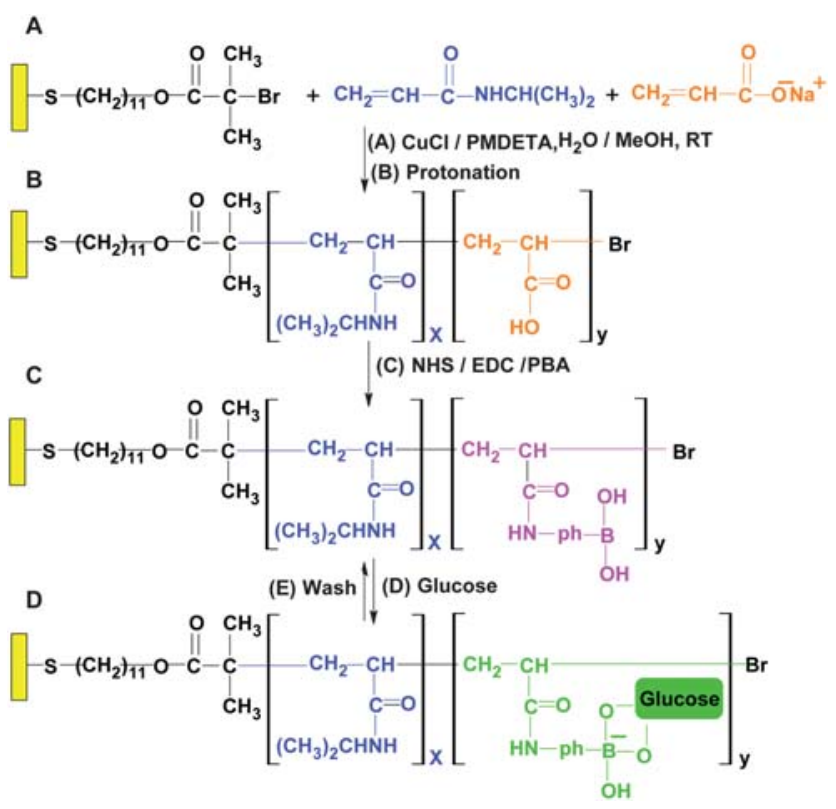

Fig. 1 Synthesis of glucose-responsive polymer brushes by combining $\mu \mathrm{CP}$, ATRP and chemical modification. (A) $\mu \mathrm{CP}$ to obtain patterned initiator layer, (B) ATRP is used to amplify the initiator layer to obtain copolymer brushes, and protonation is used to obtain - $\mathrm{COOH}$ containing brushes, (C) PNIPAAM-co-PAA-PBA brushes are obtained by conjugating the amine-terminated PBA to the carboxylic acid-terminated PNIPAAM-co-PAA, and (D) brush conformational response to glucose. (NHS: $N$-Hydroxysuccinimide, EDC: $N$-ethyl- $N$-(3-dimethylaminopropyl) carbodiimide). 
poly( $N$-isopropylacrylamide)-co-poly(sodium acrylate) (PNIPAAMco-PNaAc) brushes on gold substrates by amplifying a micro-contact printed $(\mu \mathrm{CP})$ initiator layer via surface-initiated atom transfer radical polymerization (ATRP) (Fig. 1A and B). Protonation of the polymer brush resulted in poly( $N$-isopropylacrylamide)-co-poly(acrylic acid) (PNIPAAM-co-PAA) ${ }^{23}$ We then synthesized PNIPAAM-co-PAAPBA brushes by conjugating the amine-terminated PBA to the carboxylic acid functional groups of PNIPAAM-co-PAA (Fig. 1C). ${ }^{20,21}$ We evaluated the stimulus-response of these polymer brushes to changes in glucose concentration and solution $\mathrm{pH}$ by measuring concomitant brush height changes by means of atomic force microscopy. For proof of concept we measured the relative changes of surface stress of (i) piezoresistive cantilevers on a Cantion Cantichip ${ }^{\circledR}$ that were functionalized with MPBA-SAM and PNIPAAM-co-PAA-PBA brushes, and (ii) the surface stress response of an Octosensis ${ }^{\circledR}$ (Micromotive $\mathrm{GmbH}$, Germany) micromechanical cantilever sensor array, functionalized with gold, MPBA-SAM, PNIPAAM-co-PAA and PNIPAAM-co-PAA-PBA using a multiplexed optical beam deflection setup (Scentris ${ }^{\mathrm{TM}}$, Veeco, USA) ${ }^{24}$

Contact mode atomic force microscopy (AFM) images $(40 \mu \mathrm{m} \times$ $40 \mu \mathrm{m})$ in PBS buffer at $\mathrm{pH} 9.0$ with and without glucose $(50 \mathrm{mM})$ are shown in Fig. 2A and B and reflect the situation depicted in Figs 1C
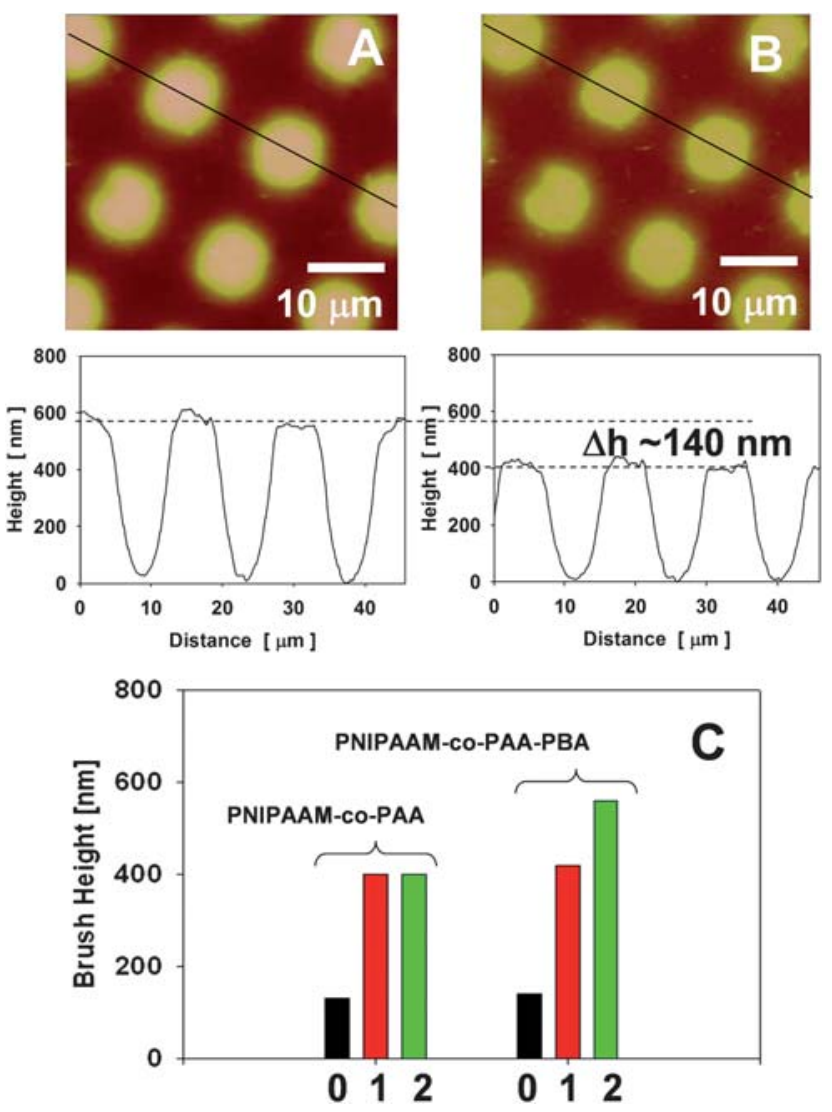

Fig. 2 Contact mode AFM height image $(40 \mu \mathrm{m} \times 40 \mu \mathrm{m})$ of PNIPAAM-co-PAA-PBA polymer brushes at room temperature in PBS buffer at $\mathrm{pH}$ 9.0. Samples were placed in a fluid cell and imaged (A) with $50 \mathrm{mM}$ glucose and (B) without glucose. (EDC: $8 \mathrm{mg} \mathrm{mL}^{-1}$, NHS: $5 \mathrm{mg} \mathrm{mL}^{-1}$, PBA: $16 \mathrm{mg} \mathrm{mL}^{-1}$ ). (C) Brush height of PNIPAAM-co-PAA and PNIPAAM-co-PAA-PBA in different conditions (0: in air, 1: in PBS buffer at pH 9.0, and 2: in PBS buffer at $\mathrm{pH} 9.0$ with $50 \mathrm{mM}$ glucose). and $\mathrm{D}$, above. When placed in PBS buffer at $\mathrm{pH} 9.0$ in the absence of glucose, both PNIPAAM-co-PAA (height $\approx 130 \mathrm{~nm}$ in air) and PNIPAAM-co-PAA-PBA ( $\sim 140 \mathrm{~nm}$ in air) brushes swell significantly, and reach heights of $400 \mathrm{~nm}$ and $420 \mathrm{~nm}$, respectively. However, when immersed in $50 \mathrm{mM}$ glucose, only the PNIPAAMco-PAA-PBA brushes swell further to reach a height of $560 \mathrm{~nm}$, while there is no additional height change for the PNIPAAM-co-PAA brushes (Fig. 2C). Soluble glucose binds to the tetrahedral, ionized boronate species within the brush and causes the observed additional swelling response. ${ }^{17,20,21}$ Brush swelling due to the complexation of glucose is likely twofold: (i) the incorporation of a hydrophilic molecule and (ii) the increase in negative charge within the brush, which increases coulombic repulsive interactions, and osmotic pressure due to the increase in counterion concentration within the brush (see also below). These results confirm PBA's ability to complex glucose and cause conformational changes of PNIPAAM-co-PAAPBA brushes.

The swelling response of the copolymer brush can be maximized by increasing the concentration of the responsive functional groups that increase the specific driving force for swelling. ${ }^{21}$ Increasing the concentration of EDC/NHS in the conjugation step provides one means of increasing the degree of substitution with PBA. Fig. 3A shows that PNIPAAM-co-PAA brushes activated with high EDC/ NHS concentrations posses also a large glucose swelling response. This is likely due to the higher degree of substitution with PBA in the brush, and the ensuing ability to complex more glucose. The swelling ratio of the PNIPAAM-co-PAA-PBA brushes can also be controlled by the $\mathrm{pH}$. $\mathrm{pH}$ affects the equilibrium between the neutral trigonal form of PBA, the charged boronate anion, and the PBA complex with glucose (Fig. 3C-E). At low pH, the neutral form of PBA is relatively hydrophobic, with increasing $\mathrm{pH}$, however, more PBA groups convert from the hydrophobic, uncharged to the hydrophilic, charged form, and the degree of swelling of the PNIPAAM-co-PAAPBA brushes increases as a result (Fig. 3D). As shown in Fig. 3A, the height of the brushes exposed to buffer without glucose is $300 \mathrm{~nm}$ at $\mathrm{pH} 5$; upon exposure to $50 \mathrm{mM}$ glucose, the height increases to $495 \mathrm{~nm}$. At $\mathrm{pH}=9$, the height of the brushes in the absence of glucose is $360 \mathrm{~nm}$, i.e., $20 \%$ higher than at $\mathrm{pH}=5$, and upon exposure to $50 \mathrm{mM}$ glucose, the brush height reaches $610 \mathrm{~nm}$, i.e., $23 \%$ higher than at $\mathrm{pH}=5$. To further investigate the responsive properties, the dependence of the glucose sensitivity for the PNIPAAM-co-PAA-PBA brush on the concentration of glucose was considered. As shown in Fig. 3A, the swelling ratio of a PNIPAAMco-PAA-PBA brush increases with increasing glucose concentration up to a concentration of $50 \mathrm{mM}$.

At a fixed $\mathrm{pH}$, an increase of glucose concentration causes a shift of the complexation equilibrium, and likely provides thus additional driving force for PBA-glucose complexation until a new equilibrium is reached. The charged form of the PBA group is further stabilized by the complexation with glucose,$^{17,20,21,25}$ giving rise to increasing anionic charge density and thus additional brush swelling (Fig. 3E). When the glucose concentration exceeds $50 \mathrm{mM}$, the glucose response curves become negatively sloped, which could be due to a charge shielding effect that affects the swelling state of polyelectrolyte brushes. More detailed studies are necessary, however, to elucidate this phenomenon. We also found that brush swelling is almost reversible, when the polymer brushes were subjected to a rinse step with PBS and subsequent $\mathrm{pH}$ adjustment (Fig. 3B). The small differences in polymer brush heights between the first and second 

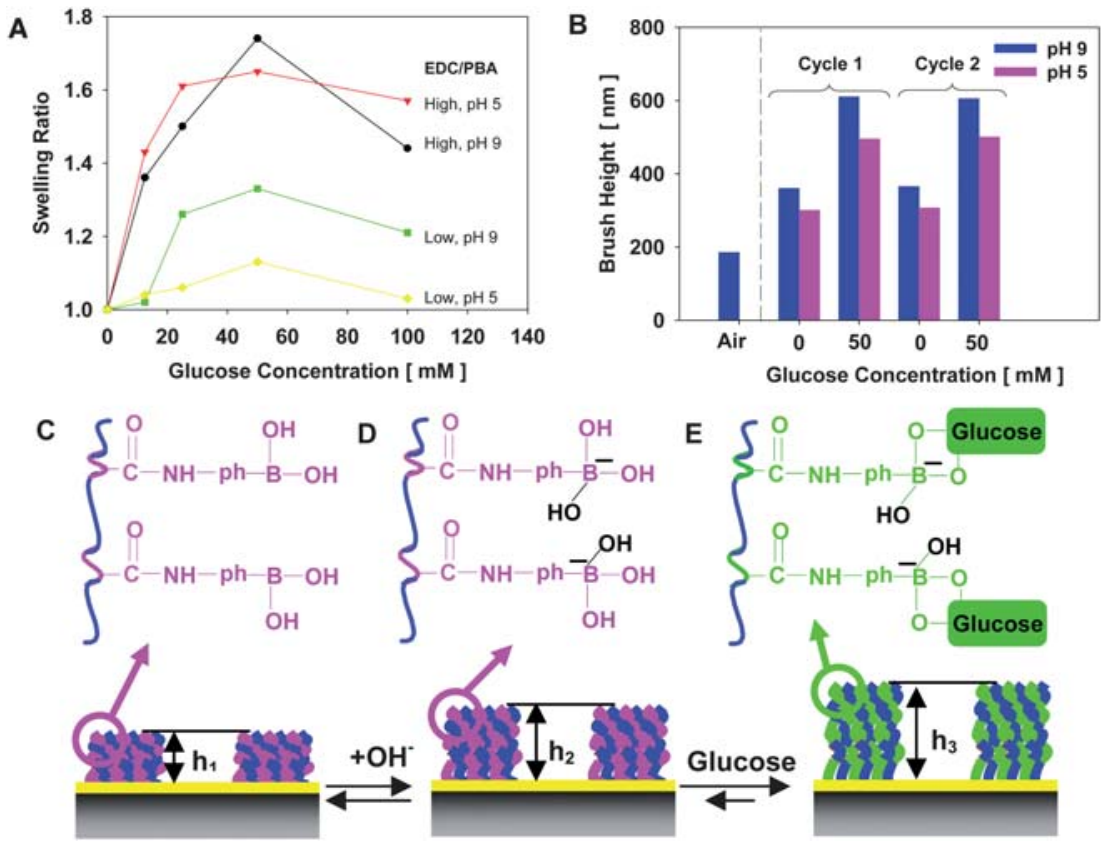

Fig. 3 (A) Equilibrium brush swelling ratio plotted as a function of glucose concentration, for brushes with high degree of PBA substitution (EDC: $100 \mathrm{mg} \mathrm{mL}^{-1}$, NHS: $62.5 \mathrm{mg} \mathrm{mL}^{-1}$, PBA: $16 \mathrm{mg} \mathrm{mL}^{-1}$ ) and low degree of PBA substitution (EDC: $8 \mathrm{mg} \mathrm{mL}^{-1}$, NHS: $5 \mathrm{mg} \mathrm{mL}^{-1}$, PBA: $16 \mathrm{mg} \mathrm{mL}^{-1}$ ) and for two $\mathrm{pH}$ values. (B) Brush height in air, in buffer and in glucose solution. Key: brushes exposed to $50 \mathrm{mM}$ glucose at $\mathrm{pH} 9.0$ (blue bar) and brushes exposed to $50 \mathrm{mM}$ glucose at pH 5.0 (purple bar). (C-E) PBA in neutral trigonal form, charged boronate anion, and complex with glucose, and ensuing brush heights $(h)$, where $h_{3}>h_{2}>h_{1}$.

cycle result in part from differences in the normal forces applied during imaging the soft brush structures.

There has been a proliferation of microcantilever-based sensors because of their ability to be operated in small sample volumes and in parallel. ${ }^{6,24,26-31}$ A micro-cantilever sensor typically consists of two lamina that differ in their physical or chemical nature so that physical adsorption, chemical reactions, or biomolecular binding events preferentially occur at one of the two surfaces, causing a differential stress that induces cantilever bending. ${ }^{32}$ Building on the encouraging results discussed above, we functionalized two types of microcantilever arrays with glucose responsive polymer brushes and explored their use in a prototypical example for the detection of glucose.

First we studied the surface stress generated by exposing PNIPAAM-co-PAA-PBA brush and 4-mercaptophenylboronic acid (MPBA)-SAM functionalized piezoresistive microcantilevers (thickness $0.5 \mu \mathrm{m}$, length $120 \mu \mathrm{m}$, width $35 \mu \mathrm{m}$, Cantion Cantichip $\left.{ }^{\circledR}\right)$ to $10 \mathrm{mM}$ glucose in PBS (pH 7.0). We found that the overall bending response and thus the surface stress is significantly larger than that for a (MPBA)-SAM functionalized microcantilever under the same solution conditions (Fig. 4), and the stress response rate (slope) for the PNIPAAM-co-PAA-PBA brush functionalized lever was about twice that for the MPBA-SAM functionalized lever. The approximate surface stress was calculated using Stoney's formula, ${ }^{33}$

$$
\sigma=\frac{E t_{\mathrm{s}}^{2}}{6(1-v)} \times \frac{2 z}{z^{2}+L^{2}}
$$

where $t_{\mathrm{s}}$ is the thickness of the cantilever, $E$ is its Young's modulus, $v$ is Poisson's ratio, and $\sigma$ is the differential surface stress between the topside and underside of the micromechanical cantilever. The deflection of the cantilever is $Z$ and its length is $L$. We note that for the piezoresistive microcantilevers used here, no inference about the deflection direction can be drawn, and the surface stress is arbitrarily plotted as positive in Fig. 4

To further study the effects of glucose binding on the bending response of micromechanical cantilevers, we performed experiments using silicon micromechanical cantilever sensor arrays (thickness $5 \mu \mathrm{m}$, length $500 \mu \mathrm{m}$, width $90 \mu \mathrm{m}$, Octosensis $\left.{ }^{\circledR}\right)$. The deflection of the micromechanical cantilevers was measured by means of a multiplexed beam deflection setup. The eight cantilevers on the cantilever array were divided into four groups (two adjacent levers in each group) where each group had a different coating on the cantilever top surface (Fig. 5). For the analysis, the deflection of the two cantilevers in any one group was averaged. All cantilevers were first coated with a $50 \mathrm{~nm}$ gold film. Levers in group I were not further treated, levers in

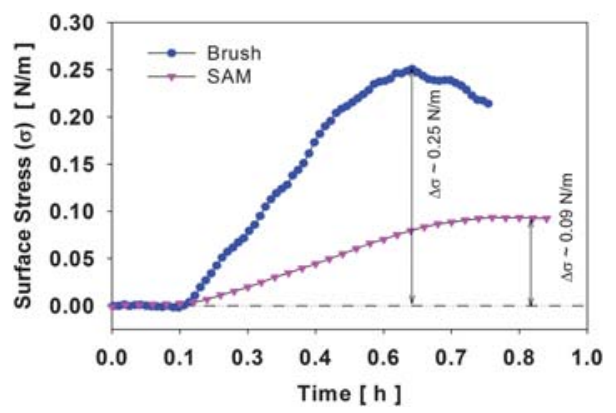

Fig. 4 Relative magnitude of the surface stress response of piezoresistive cantilevers (Cantion Cantichip ${ }^{\circledR}$ ) plotted as a function of time, in response to injection of $10 \mathrm{mM}$ glucose solution (PBS, $\mathrm{pH}$ 7.0). Results for a cantilever functionalized with an MPBA-SAM and the averaged response from three cantilevers, functionalized with PNIPAAM-coPAA-PBA brushes are shown. 


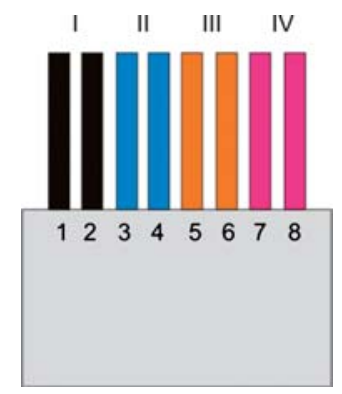

Fig. 5 Schematic topside view of the micromechanical cantilever array. Cantilevers of the first group are coated with gold (I), of the second group (II) with MPBA-SAM, of the third group (III) with PNIPAAM-co-PAA, and of the fourth group (IV) with PNIPAAM-co-PAA-PBA.

group II were coated with MPBA-SAM, levers in group III with PNIPAAM-co-PAA, and levers in group IV with PNIPAAM-coPAA-PBA. Once mounted into a flow cell, deflection measurements were started immediately after injection of PBS buffer solution. A $50 \mathrm{mM}$ solution of glucose in PBS buffer was injected once the cantilevers reached an equilibrium deflection in PBS. Measurements were made at two different $\mathrm{pH}$ values. The averaged deflection response of the cantilevers in response to the solvent conditions (PBS buffer and $50 \mathrm{mM}$ glucose in PBS) at two different $\mathrm{pH}$ conditions is plotted as a function of time in Fig. 6.

First we discuss the effect of $\mathrm{pH}$ on cantilever deflection in the absence of glucose. Changing the $\mathrm{pH}$ from 5 to 9 , the deflection response of cantilevers coated with gold (group I) and the MPBASAM (group II) changed little, $11 \mathrm{~nm}$ and $<1 \mathrm{~nm}$, respectively. However, the cantilevers coated with PNIPAMM-co-PAA (group III) and PNIPAMM-co-PAA-PBA (group IV) bend away from the polymer brushes by $208 \mathrm{~nm}$ and $280 \mathrm{~nm}$. The corresponding surface stress change was calculated to be $1.26 \mathrm{~N} \mathrm{~m}^{-1}$ and $1.69 \mathrm{~N} \mathrm{~m}^{-1}$, respectively. At $\mathrm{pH} 9$ both $\mathrm{PAA}$ and $\mathrm{PBA}$ are ionized and the repulsive interaction of charges caused by the increase of the ion concentration in the polymer chains leads to an increase in swelling, in agreement with the AFM height measurements discussed above. ${ }^{34}$

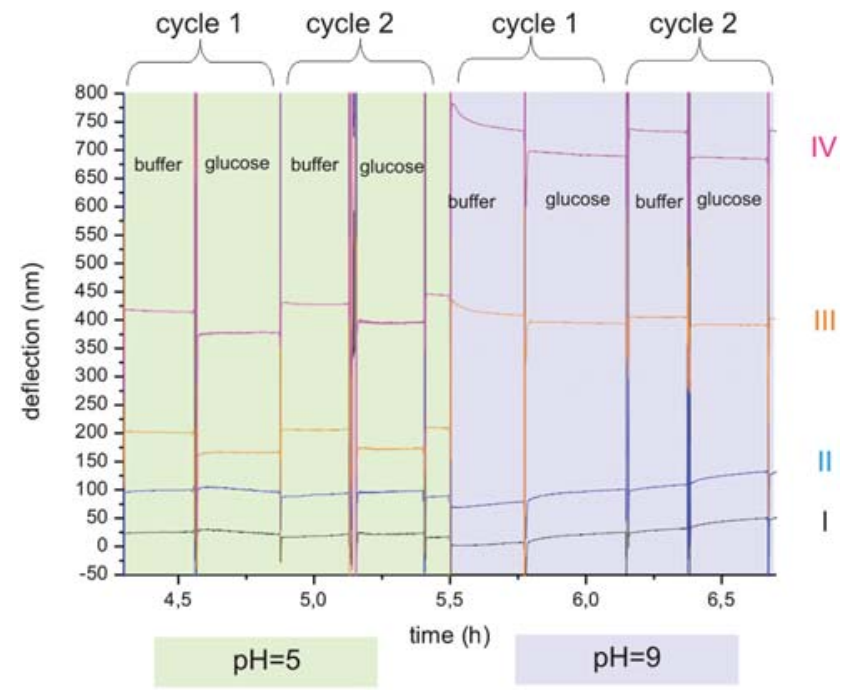

Fig. 6 Average deflection response of the micromechanical cantilevers as schematically shown in Fig. 5 at different solution conditions and at two $\mathrm{pH}$ values.

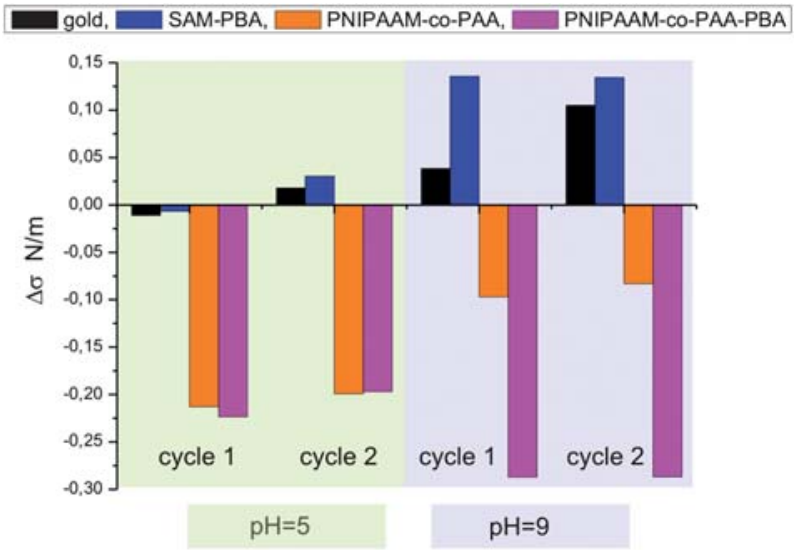

Fig. 7 Surface stress response upon exposure to $50 \mathrm{mM}$ glucose in PBS at $\mathrm{pH}=5$ and $\mathrm{pH}=9$. Black column (group I): cantilevers coated with gold; blue column (group II): cantilevers coated with PBA-SAM; orange column (group III): cantilevers coated with PNIPAAM-co-PAA; purple column (group IV): cantilevers coated with PNIPAAM-co-PAA-PBA.

Next we studied the effect of exposure to $50 \mathrm{mM}$ glucose on the cantilever bending response at both $\mathrm{pH}$ values. The cantilevers coated with PNIPAMM-co-PAA (group III) and PNIPAMM-co-PAAPBA (group IV) bend towards the polymer brushes upon injecting glucose solution in the cell. As seen in Fig. 6, the cantilever bending response is reversible upon sequentially changing the solvent conditions between PBS buffer and $50 \mathrm{mM}$ glucose in PBS at both $\mathrm{pH}$ values. To obtain geometry independent data, we again calculate the surface stress change of the cantilevers using eqn (1); results are shown in Fig. 7. At pH 5, the surface stress change of the cantilevers coated with gold (group I) and MPBA-SAM (group II) is small, indicating no significant glucose adsorption. The bending of the cantilevers coated with PNIPAMM-co-PAA (group III) and with PNIPAMM-co-PAA-PBA (group IV) relaxes, with a surface stress decrease of about $0.20 \mathrm{~N} \mathrm{~m}^{-1}$. Since both cantilevers respond about equally, there is no obvious evidence of specific binding of glucose to PNIPAMM-co-PAA-PBA.

At $\mathrm{pH}$ 9, the deflection of cantilevers coated with gold (group I) and MPBA-SAM (group II) bends away from the coated side with a concomitant surface stress change of $0.075 \mathrm{~N} \mathrm{~m}^{-1}$ and $0.14 \mathrm{~N} \mathrm{~m}^{-1}$, respectively. The bending of the cantilevers coated with PNIPAMMco-PAA (group III) and with PNIPAMM-co-PAA-PBA (group IV) again relaxed, but now the surface stress decreased by $0.09 \mathrm{~N} \mathrm{~m}^{-1}$ and by $0.27 \mathrm{~N} \mathrm{~m}^{-1}$, respectively. The apparent bending difference and the concomitant change of surface stress between these two cantilevers indicate a specific binding of glucose to PNIPAMM-co-PAA-PBA. Furthermore, as already seen in Fig. 4, the magnitude of the surface stress change of the PNIPAMM-co-PAA-PBA coated cantilevers is about twice as large as that of cantilevers coated with MPBA-SAM.

In summary, we have shown the synthesis of glucose-responsive PNIPAAM-co-PAA-PBA brushes, and explored their use in a prototypical example for their potential as polymer brushes-functionalized microcantilevers for the detection of glucose. We evaluated the stimulus-response of the polymer brushes to changes with glucose concentration and solution $\mathrm{pH}$ by measuring concomitant brush height changes. Glucose-responsive PNIPAAM-co-PAA-PBA brushes show a large, reversible swelling response in the presence of free glucose at physiologically relevant concentrations. The deflection 
and surface stress response of micromechanical cantilevers, functionalized with PNIPAMM-co-PAA-PBA brushes, are substantially larger and faster than that for MPBA-SAM functionalized levers. This shows the promise of PNIPAAM-co-PAA-PBA brushes for micromechanical cantilever glucose sensing applications, and demonstrates, more generally, the potential of responsive polymer brushes to sense and transduce changes in a solution environment efficiently.

\section{Acknowledgements}

S. Z. thanks the National Science Foundation for support through grants NSF DMR-0502953 and NSF NIRT CBET-0609265. R. B. and T. L. thank the DFG-Sonderforschungsbereich 625 "From Single Molecules to Nanoscopically Structured Materials" for support.

\section{Notes and references}

1 G. G. Bumbu, G. Kircher, M. Wolkenhauer, R. Berger and J. S. Gutmann, Macromol. Chem. Phys., 2004, 205, 1713-1720.

2 F. Zhou, W. M. Shu, M. E. Welland and W. T. S. Huck, J. Am. Chem. Soc., 2006, 128, 5326-5327.

3 N. I. Abu-Lail, M. Kaholek, B. LaMattina, R. L. Clark and S. Zauscher, Sens. Actuators, B, 2006, 114, 371-378.

4 A. Valiaev, N. I. Abu-Lail, D. W. Lim, A. Chilkoti and S. Zauscher, Langmuir, 2007, 23, 339-344.

5 F. Zhou, P. M. Biesheuvel, E. Y. Chol, W. Shu, R. Poetes, U. Steiner and W. T. S. Huck, Nano Lett., 2008, 8, 725-730.

6 S. Singamaneni, M. C. LeMieux, H. P. Lang, C. Gerber, Y. Lam, S. Zauscher, P. G. Datskos, N. V. Lavrik, H. Jiang, R. R. Naik, T. J. Bunning and V. V. Tsukruk, Adv. Mater., 2008, 20, 653-680.

7 C. Bradley, N. Jalili, S. K. Nett, L. Q. Chu, R. Forch, J. S. Gutmann and R. Berger, Macromol. Chem. Phys., 2009, 210, 1339-1345.

8 R. B. Fong, Z. L. Ding, C. J. Long, A. S. Hoffman and P. S. Stayton, Bioconjugate Chem., 1999, 10, 720-725.

9 Q. He, A. Kueller, S. Schilp, F. Leisten, H. A. Kolb, M. Grunze and J. B. Li, Small, 2007, 3, 1860-1865.

10 T. Chen, J. M. Zhang, D. P. Chang, A. Garcia and S. Zauscher, $A d v$. Mater., 2009, 21, 1825-1829.

11 S. J. Ahn, M. Kaholek, W. K. Lee, B. LaMattina, T. H. LaBean and S. Zauscher, Adv. Mater., 2004, 16, 2141-2145.
12 X. Yin, A. S. Hoffman and P. S. Stayton, Biomacromolecules, 2006, 7, 1381-1385.

13 E. S. Forzani, H. Q. Zhang, L. A. Nagahara, I. Amlani, R. Tsui and N. J. Tao, Nano Lett., 2004, 4, 1785-1788.

14 J. Wang, Chem. Rev., 2008, 108, 814-825.

15 K. Kataoka, H. Miyazaki, M. Bunya, T. Okano and Y. Sakurai, J. Am. Chem. Soc., 1998, 120, 12694-12695.

16 A. Matsumoto, S. Ikeda, A. Harada and K. Kataoka, Biomacromolecules, 2003, 4, 1410-1416.

17 F. Xia, H. Ge, Y. Hou, T. L. Sun, L. Chen, G. Z. Zhang and L. Jiang, Adv. Mater., 2007, 19, 2520-2524.

18 S. Kitano, K. Kataoka, Y. Koyama, T. Okano and Y. Sakurai, Makromol. Chem., Rapid Commun., 1991, 12, 227-233.

19 B. Elmas, M. A. Onur, S. Senel and A. Tuncel, Colloid Polym. Sci., 2002, 280, 1137-1146.

20 T. Hoare and R. Pelton, Macromolecules, 2007, 40, 670-678.

21 A. Matsumoto, R. Yoshida and K. Kataoka, Biomacromolecules, 2004, 5, 1038-1045.

22 D. Shiino, K. Kataoka, Y. Koyama, M. Yokoyama, T. Okano and Y. Sakurai, J. Controlled Release, 1994, 28, 317-318.

23 R. Dong, S. Krishnan, B. A. Baird, M. Lindau and C. K. Ober, Biomacromolecules, 2007, 8, 3082-3092.

24 H. P. Lang, R. Berger, C. Andreoli, J. Brugger, M. Despont, P. Vettiger, C. Gerber, J. K. Gimzewski, J. P. Ramseyer, E. Meyer and H. J. Guntherodt, Appl. Phys. Lett., 1998, 72, 383-385.

25 Y. J. Zhang, Y. Guan and S. Q. Zhou, Biomacromolecules, 2007, 8, 3842-3847.

26 R. Berger, C. Gerber, J. K. Gimzewski, E. Meyer and H. J. Guntherodt, Appl. Phys. Lett., 1996, 69, 40-42.

27 J. Fritz, M. K. Baller, H. P. Lang, H. Rothuizen, P. Vettiger, E. Meyer, H. J. Guntherodt, C. Gerber and J. K. Gimzewski, Science, 2000, 288, 316-318.

28 H. F. Ji, E. Finot, R. Dabestani, T. Thundat, G. M. Brown and P. F. Britt, Chem. Commun., 2000, 457-458.

29 J. H. Pei, F. Tian and T. Thundat, Anal. Chem., 2004, 76, 292-297.

30 L. A. Pinnaduwage, A. Gehl, D. L. Hedden, G. Muralidharan, T. Thundat, R. T. Lareau, T. Sulchek, L. Manning, B. Rogers, M. Jones and J. D. Adams, Nature, 2003, 425, 474-474.

31 R. Zhang, A. Best, R. Berger, S. Cherian, S. Lorenzoni, E. Macis, R. Raiteri and R. Cain, Rev. Sci. Instrum., 2007, 78, 7.

32 G. H. Wu, H. F. Ji, K. Hansen, T. Thundat, R. Datar, R. Cote, M. F. Hagan, A. K. Chakraborty and A. Majumdar, Proc. Natl. Acad. Sci. U. S. A., 2001, 98, 1560-1564.

33 R. Raiteri and H. J. Butt, J. Phys. Chem., 1995, 99, 15728-15732.

34 G. Gerald, G. Margarita, S. Gunnar, S. Joerg, A. Karl-Friedrich and R. Andreas, Macromol. Symp., 2004, 210, 403-410. 